Ann. Zootech., I971, 20 (4), 427-435.

\author{
I.N.R.A. \\ BIBLIOTHEQUE UO 3E9:
DOMAINE DE CROUELLE \\ 63039 \\ CLERMONT-FD CEDEX?
}

\title{
L'INDICE DE FIBROSITÉ DES FOURRAGES : SA SIGNIFICATION ET SON UTILISATION POUR LA PRÉVISION DE LA VALEUR ALIMENTAIRE DES FOURRAGES
}

\author{
M. CHENOST (1) et Élisabeth GRENET \\ avec la collaboration technique de Marie JAILler \\ Station de Recherches sur l'Élevage des Ruminants, \\ Centre de Recherches de Clermont-Ferrand, I. N. R. A., \\ 63 - Saint-Genès-Champanelle
}

\section{RÉSUMÉ}

L'indice de fibrosité des fourrages, dont la méthode de mesure a été mise au point antérieurement (Chenost, 1966), reflète leur composition morphologique (pourcentage de feuilles), leur composition chimique (proportion de constituants membranaires) et leur aptitude à être réduits en fines particules dans le rumen (digestibilité en sachet, temps passé par l'animal à mastiquer).

Le fait d'associer à l'indice de fibrosité un autre critère simple de la prévision de la valeur nutritive et alimentaire des fourrages, tel que le pourcentage de feuilles ou la teneur en cellulose Weende, permet d'améliorer la précision des résultats obtenus avec l'indice de fibrosité seul ou à l'aide de ces critères considérés séparément.

Nous avons calculé à partir de 152 fourrages, de famille botanique et de cycles végétatifs différents, les équations de régression liant la digestibilité, la quantité de matière sèche ingérée et la quantité de matière organique digestible ingérée à l'ensemble indice de fibrosité-cellulose Weende. On améliore les liaisons en considérant séparément les graminées du rer cycle et l'ensemble des graminées.

\section{INTRODUCTION}

Dans un travail précédent (CHENOST, I966) nous avons essayé de relier la valeur alimentaire des fourrages à leurs caractéristiques physiques. Nous avons mis en évidence, ainsi que Troelsen et BIGSBY (I964) et TroklsEn et al. (1969, r970), que l'aptitude d'un fourrage à être réduit en fines particules après une action mécanique est en

( $\left.{ }^{1}\right)$ Adresse actuelle : Station de Recherches zootechniques, Centre de Recherches agronomiques des Antilles et de la Guyane, Domaine Duclos, Petit-Bourg (Guadeloupe). 
liaison étroite avec la quantité qui peut en être ingérée par le ruminant. Nous avions trouvé une bonne relation entre la digestibilité ou les quantités ingérées d'une part et l'indice de fibrosité défini par l'énergie électrique dépensée pour broyer une prise de $5 \mathrm{~g}$ de fourrage d'autre part. Nous avons voulu voir s'il était possible de préciser la signification de l'indice de fibrosité et d'améliorer la prévision de la valeur alimentaire, en lui associant d'autres critères simples, tels que la teneur en matières azotées, en cellulose brute ou en lignocellulose, la composition morphologique ou la digestibilité in vitro ou en sachets de nylon.

\title{
MATÉRIEL E'T MÉTHODES
}

\author{
Fourrages
}

Nous avons utilisé :

- d’une part : 34 échantillons de fourrages verts de graminées récoltés au $\mathbf{I}$ er cycle de croissance dont nous connaissions la composition chimique (constituants membranaires, cellulose brute, matières azotées et cendres), la composition morphologique, la digestibilité et les quantités ingérées par le mouton.

- d'autre part : 152 échantillons de foins et fourrages verts de graminées et légumineuses dont nous connaissions la digestibilité et la quantité ingérée par le mouton et les teneurs en cellulose brute, matières azotées et cendres.

\section{Méthodes}

Nous avons mesuré l'indice de fibrosité de tous les échantillons selon la technique décrite antérieurement (Chenost, I966) mais nous avons utilisé un wattmètre (Guerpillon) différent; l'unité d'indice de fibrosité n'est plus $\mathrm{r} / \mathrm{r} O \mathrm{wh}$ mais $0,2928 \frac{\mathrm{wh}}{\mathrm{IO}}$.

Nous avons soumis le I er groupe des 34 échantillons de fourrages verts au test de digestion dans le rumen par la méthode des sachets de nylon (Demarguilly et Chenost, r969; Chenost et al., r97o), au test de digestion in vitvo par la méthode de TiLle y et TERRY (r963) et à l'attaque par la cellulase (JARrige et Thivend, I969; JARRige et al., 1970). Nous avons dosé, en outre, leur teneur en membranes, cellulose vraie, hémicelluloses et lignine par la méthode de fractionnement glucidique de JARRIGE (r96I) ainsi que la lignocellulose (ADF de VAN SoEST, Ig63).

Pour $\mathrm{I}_{3}$ de ces fourrages, nous avons mesuré par enregistrement des mouvements de la mâchoire selon la méthode de Ruckebusch et Marguet (I963) les temps d'ingestion, de rumination et de mastication (ingestion + rumination) des moutons que nous avons exprimés en $\mathrm{mn}$ par $\mathrm{kg}$ de matière sèche ingérée.

\section{Abréviations utilisées}

Dans les tableaux, nous utiliserons les abréviations suivantes :

IF Indice de fibrosité

CB Cellulose brute Weende

MAT Matières azotées totales

ADF Lignocellulose de VAN SoEsT

DIV Digestibilité in vitro (p. 10o)

CUD sachets Digestibilité in vivo (p. Ioo) par la méthode des sachets de nylon

F Pourcentage de feuilles du fourrage

$\mathrm{T}+\mathrm{E}$ Pourcentage de "tiges + épis" du fourrage 


\section{RÉSULTATS ET DISCUSSION}

\section{Signification de l'indice de fibrosité}

Nous avons calculé les coefficients de corrélation simples et multiples permettant de mettre en parallèle l'indice de fibrosité avec les différentes caractéristiques des 34 fourrages, prises seules ou associées par 2 ou par 3 (tabl. I).

Les meilleures liaisons sont obtenues avec la proportion de feuilles $(-0,863)$, la teneur en cellulose Weende $(+0,8 \mathrm{I} 2)$, la teneur en membranes $(+0,836), 1$ 'ADF $(+0,823)$ et la teneur en lignocellulose $(+0,777)$; elles montrent que l'indice de fibrosité d'un fourrage augmente avec la proportion de tiges et la teneur en constituants membranaires.

Le fait d'associer la proportion de feuilles aux critères membranaires, notamment à 1 'ADF et à la teneur en membranes, permet d'augmenter la valeur des coefficients de corrélation, $\mathrm{R}=0,928$ et $\mathrm{R}=0,922$ respectivement; il en est de même lorsque nous associons la teneur en matières azotées totales (critère du contenu cellulaire) à la teneur en cellulose Weende ou à la teneur en membranes. L e coefficient de corrélation le plus élevé entre l'indice de fibrosité et deux variables est obtenu avec le pourcentage de feuilles et la digestibilité en sachet pendant 48 heures $(R=0,945)$.

Le fait d'ajouter une troisième variable, telle que le temps d'ingestion ou de rumination ou la digestibilité en sachets pendant 48 heures, aux critères membranaires et morphologiques permet d'augmenter encore la valeur des coefficients de corrélation (tabl. I). Ceci est normal car l'indice de fibrosité est un travail (énergie électrique) qui dépend :

- de la résistance du fourrage au broyage, qui augmente avec sa teneur en membranes et son pourcentage de tiges;

- du temps nécessaire pour réduire le fourrage en fines particules : il résulte chez l'animal de l'action conjuguée des microorganismes du rumen et de la mastication merycique qui peuvent s'apprécier au travers de la digestibilité en sachets et du temps passé par l'animal à mastiquer par $\mathrm{kg}$ de matière sèche ingérée.

Pour 3 variables, le coefficient de corrélation le plus élevé est cependant obtenu avec la teneur en matières azotées totales, le temps d'ingestion et la digestibilité sachets 48 heures $(R=0,969)$.

En résumé, 1'indice de fibrosité reflète non seulement la composition morphologique et chimique du fourrage mais également son aptitude à être réduit en fines particules.

\section{Prévision de la valeur alimentaire}

I. Pour les 34 fourrages précédents, nous avons comparé la précision de la prévision (déviation de la régression) de la digestibilité et des quantités de matière sèche et de matière organique digestible ingérées obtenue à partir de l'indice de fibrosité et à partir des différentes caractéristiques de ces fourrages (tab1. 2). Nous avons aussi cherché à améliorer la précision en associant un second critère à l'indice de fibrosité (tabl. 2).

L'indice de fibrosité permet de prévoir la digestibilité, la quantité de matière 
TABLEAU I

Signification de l'indice de fibrosité: liaisons entre l'indice de fibrosité et les différentes caractéristiques du fourrage considérées seules, deux à deux, trois à trois

\begin{tabular}{|c|c|c|c|c|}
\hline \multicolumn{3}{|c|}{ Critères explicatifs } & \multirow{2}{*}{$\begin{array}{c}\text { Nombre } \\
\text { d'échantillons }\end{array}$} & \multirow{2}{*}{$\begin{array}{l}\text { Coefficient de } \\
\text { corrélation } \\
r \text { ou } \mathrm{R}\end{array}$} \\
\hline fer critère & $2^{\mathrm{e}}$ critère & $3^{e}$ critère & & \\
\hline Cellulose Weende & & & $3{ }^{\prime}$ & $+0,813$ \\
\hline Cellulose Weende & Cendres & & 34 & 0,877 \\
\hline Cellulose Weende & Cendres & DIV $8 \mathrm{~h}$ & 34 & 0,942 \\
\hline Cellulose Weende & MAT & & 34 & 0,927 \\
\hline Cellulose Weende & MAT & $\begin{array}{l}\text { Temps ingestion } \\
+ \text { rumination }\end{array}$ & 13 & 0,936 \\
\hline Cellulose Weende & $\begin{array}{l}\text { Pourceutage } \\
\text { de feuilles }\end{array}$ & & 31 & 0,907 \\
\hline Cellulose Weende & $\begin{array}{l}\text { Pourcentage } \\
\text { de feuilles }\end{array}$ & Sachets $48 \mathrm{~h}$ & 31 & 0,951 \\
\hline Membranes & & & 34 & $+0,836$ \\
\hline Membranes & Cendres & & $33_{t}^{\prime}$ & 0,898 \\
\hline Membranes & MAT & & $3^{\prime}$ & 0,927 \\
\hline Membranes & $\begin{array}{l}\text { Pourcentage } \\
\text { de feuilles }\end{array}$ & & 13 & 0,922 \\
\hline Membranes & $\begin{array}{l}\text { Pourcentage } \\
\text { de feuilles }\end{array}$ & $\mathrm{ADF}$ & 34 & 0,932 \\
\hline Membranes & $\begin{array}{l}\text { Pourcentage } \\
\text { de feuilles }\end{array}$ & Sachets $48 \mathrm{~h}$ & $3 q$ & 0,947 \\
\hline Lignocellulose & & & 34 & $+0,777$ \\
\hline Lignocellulose & $\begin{array}{l}\text { Pourcentage } \\
\text { de feuilles }\end{array}$ & & 31 & 0,902 \\
\hline Lignocellulose & $\begin{array}{l}\text { Pourcentage } \\
\text { de feuilles }\end{array}$ & Sachets $48 \mathrm{~h}$ & 31 & 0,949 \\
\hline Lignocellulose & Cendres & & 34 & 0,890 \\
\hline $\mathrm{ADF}$ & & & $3 / 4$ & $+0,823$ \\
\hline $\mathrm{ADF}$ & Cendres & & $3{ }^{\prime}$ & 0,905 \\
\hline $\mathrm{ADF}$ & Cendres & $\begin{array}{l}\text { Pourcentage } \\
\text { de feuilles }\end{array}$ & 31 & 0,936 \\
\hline ADF & $\begin{array}{l}\text { Pourcentage } \\
\text { de feuilles }\end{array}$ & & 31 & 0,928 \\
\hline $\mathrm{ADF}$ & $\begin{array}{l}\text { Pourcentage } \\
\text { de feuilles }\end{array}$ & Hémicellulose & 31 & 0,931 \\
\hline $\mathrm{ADF}$ & $\begin{array}{l}\text { Pourcentage } \\
\text { de feuilles }\end{array}$ & Sachets $48 \mathrm{~h}$ & 31 & 0,955 \\
\hline $\mathrm{ADF}$ & $\begin{array}{l}\text { Pourcentage } \\
\text { de feuilles }\end{array}$ & MAT & 31 & 09,33 \\
\hline $\begin{array}{l}\text { Cellulose } \\
\text { Cellulose }\end{array}$ & MAT & & $\begin{array}{l}34 \\
34\end{array}$ & $\begin{array}{r}0,804 \\
0,898\end{array}$ \\
\hline Cellulose & $\begin{array}{l}\text { Pourcentage } \\
\text { Tige }+ \text { épis }\end{array}$ & & 31 & 0,874 \\
\hline Cellulose & $\begin{array}{l}\text { Pourcentage } \\
\text { Tige }+ \text { épis }\end{array}$ & Sachet $48 \mathrm{~h}$ & 31 & 0,940 \\
\hline $\begin{array}{l}\text { Pourcentage } \\
\text { de feuilles }\end{array}$ & & & 31 & $-0,863$ \\
\hline
\end{tabular}




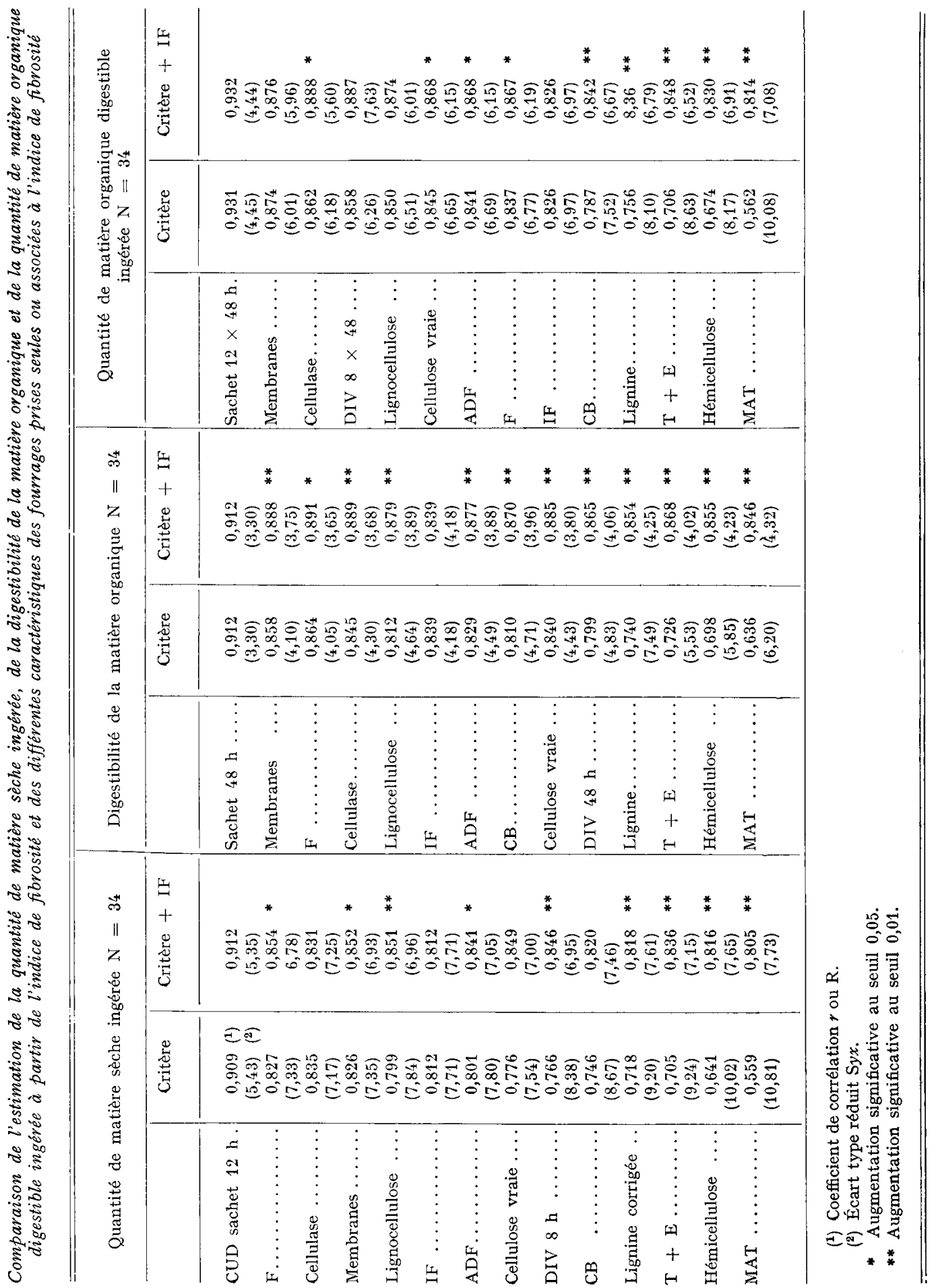


sèche ingérée et la quantité de matière organique digestible ingérée de façon plus précise que les critères usuels tels que la teneur en cellulose brute et la teneur en matières azotées. Cette précision est cependant inférieure à celle permise par la digestion par la cellulase, la digestibilité en sachet ou la digestibilité in vitro.

Lorsque nous associons l'indice de fibrosité aux différents critères de prévision de la valeur alimentaire des fourrages, la précision de la prévision s'améliore d'autant plus qu'elle était faible avec ces critères. Ainsi pour des laboratoires équipés pour les analyses chimiques simples ou encore pour les stations d'essai qui peuvent mesurer la composition morphologique des fourrages, l'association de l'indice de fibrosité, aux déterminations courantes de la teneur en cellulose Weende et en matières azotées ou de la proportion de feuilles, permet d'augmenter de façon intéressante la précision de la prévision de la valeur nutritive et alimentaire des fourrages.

\section{TABLEAU 3}

Prévision de la valeur alimentaire de 152 fourrages à partir de la teneur en cellulose Weende et de l'indice de fibrosité seul ou associé à la teneur en cellulose brute ou à la teneur en matières azotées totales

\begin{tabular}{|c|c|c|c|c|c|c|c|c|}
\hline \multirow[t]{2}{*}{$\begin{array}{c}\text { Nature } \\
\text { des fourrages }\end{array}$} & \multirow[t]{2}{*}{$\begin{array}{c}\text { Nombre } \\
\text { d'échantillons }\end{array}$} & \multirow[t]{2}{*}{$\begin{array}{c}\text { Critère } \\
\text { utilisé }\end{array}$} & \multicolumn{2}{|c|}{$\begin{array}{c}\text { Quantité } \\
\text { de matière } \\
\text { sèche ingérée }\end{array}$} & \multicolumn{2}{|c|}{$\begin{array}{c}\text { Digestibilité } \\
\text { de la } \\
\text { matière organique }\end{array}$} & \multicolumn{2}{|c|}{$\begin{array}{c}\text { Quantité } \\
\text { de matière } \\
\text { organique } \\
\text { digestible ingérée }\end{array}$} \\
\hline & & & $\mathrm{R}$ & $S(y x)$ & $\mathrm{R}$ & $S(y x)$ & $\mathrm{R}$ & $\mathrm{S}(y x)$ \\
\hline $\begin{array}{l}\text { Graminées } \\
\text { 1 er cycle }^{\text {er }}\end{array}$ & 95 & $\begin{array}{l}\text { IF } \\
\text { CB } \\
\text { MAT } \\
\text { IF, CB } \\
\text { IF, MAT }\end{array}$ & $\begin{array}{l}0,798 \\
0,842 \\
0,624 \\
0,864 \\
0,791\end{array}$ & $\begin{array}{r}8,36 \\
7,35 \\
10,83 \\
6,99 \\
8,48\end{array}$ & $\begin{array}{l}0,843 \\
0,869 \\
0,663 \\
0,893 \\
0,831\end{array}$ & $\begin{array}{l}4,65 \\
4,27 \\
6,47 \\
3,88 \\
4,81\end{array}$ & $\begin{array}{l}0,833 \\
0,875 \\
0,638 \\
0,893 \\
0,817\end{array}$ & $\begin{array}{l}6,78 \\
5,98 \\
9,45 \\
5,52 \\
7,07\end{array}$ \\
\hline $\begin{array}{l}\text { Graminées } \\
\text { repousses }\end{array}$ & 33 & $\begin{array}{l}\text { IF } \\
\mathrm{CB} \\
\text { MAT } \\
\text { IF, CB } \\
\text { IF, MAT }\end{array}$ & $\begin{array}{l}0,411 \\
0,385 \\
0,691 \\
0,375 \\
0,271\end{array}$ & $\begin{array}{r}9,57 \\
9,69 \\
7,58 \\
9,73 \\
10,10\end{array}$ & $\begin{array}{l}0,743 \\
0,657 \\
0,545 \\
0,727 \\
0,758\end{array}$ & $\begin{array}{l}4,54 \\
5,12 \\
5,70 \\
4,67 \\
4,43\end{array}$ & $\begin{array}{l}0,670 \\
0,642 \\
0,650 \\
0,629 \\
0,572\end{array}$ & $\begin{array}{l}5,61 \\
5,79 \\
5,74 \\
5,88 \\
6,20\end{array}$ \\
\hline $\begin{array}{l}\text { Ensemble } \\
\text { des } \\
\text { Graminées }\end{array}$ & 128 & $\begin{array}{l}\text { IF } \\
\text { CB } \\
\text { MAT } \\
\text { IF, CB } \\
\text { IF, MAT }\end{array}$ & $\begin{array}{l}0,732 \\
0,783 \\
0,547 \\
0,796 \\
0,726\end{array}$ & $\begin{array}{r}8,94 \\
8,15 \\
10,98 \\
7,94 \\
9,02\end{array}$ & $\begin{array}{l}0,822 \\
0,834 \\
0,637 \\
0,861 \\
0,817\end{array}$ & $\begin{array}{l}4,64 \\
4,49 \\
6,29 \\
4,14 \\
4,70\end{array}$ & $\begin{array}{l}0,807 \\
0,843 \\
0,594 \\
0,862 \\
0,794\end{array}$ & $\begin{array}{l}6,64 \\
6,04 \\
9,04 \\
5,70 \\
6,84\end{array}$ \\
\hline Légumineuses & $2 \prime$ & $\begin{array}{l}\text { IF } \\
\text { CB } \\
\text { MAT } \\
\text { IF, CB } \\
\text { IF, MAT }\end{array}$ & $\begin{array}{l}0,676 \\
0,677 \\
0,687 \\
0,718 \\
0,715\end{array}$ & $\begin{array}{r}10,58 \\
10,56 \\
10,43 \\
9,99 \\
10,03\end{array}$ & $\begin{array}{l}0,862 \\
0,830 \\
0,881 \\
0,829 \\
0,863\end{array}$ & $\begin{array}{l}3,36 \\
3,69 \\
3,13 \\
3,71 \\
3,35\end{array}$ & $\begin{array}{l}0,775 \\
0,752 \\
0,796 \\
0,782 \\
0,796\end{array}$ & $\begin{array}{l}7,33 \\
7,66 \\
7,02 \\
7,24 \\
7,02\end{array}$ \\
\hline $\begin{array}{l}\text { Ensemble } \\
\text { des } \\
\text { fourrages }\end{array}$ & 152 & $\begin{array}{l}\text { IF } \\
\text { CB } \\
\text { MAT } \\
\text { IF, CB } \\
\text { IF, MAT }\end{array}$ & $\begin{array}{l}0,715 \\
0,699 \\
0,504 \\
0,746 \\
0,719\end{array}$ & $\begin{array}{r}9,25 \\
9,44 \\
11,40 \\
8,79 \\
9,17\end{array}$ & $\begin{array}{l}0,851 \\
0,838 \\
0,407 \\
0,864 \\
0,764\end{array}$ & $\begin{array}{l}4,29 \\
4,50 \\
7,51 \\
4,14 \\
5,30\end{array}$ & $\begin{array}{l}0,802 \\
0,806 \\
0,476 \\
0,843 \\
0,774\end{array}$ & $\begin{array}{l}6,71 \\
6,67 \\
9,87 \\
6,05 \\
7,11\end{array}$ \\
\hline
\end{tabular}


2. Sur le groupe des 152 fourrages, nous avons comparé la précision des prévisions des quantités ingérées et de la digestibilité à partir de l'indice de fibrosité, de la teneur en cellulose Weende, de la teneur en matières azotées et à partir des couples indice de fibrosité-cellulose Weende et indice de fibrosité-matières azotées (tabl. 3).

L'indice de fibrosité n'apporte pas toujours une amélioration par rapport à la teneur en cellulose Weende; il en apporte cependant une par rapport à la teneur en matières azotées. On améliore la précision de la prévision de la digestibilité et surtout des quantités ingérées permise par l'indice de fibrosité, la teneur en cellulose Weende ou la teneur en matières azotées avec le couple indice de fibrosité-matières azotées et surtout indice de fibrosité-cellulose Weende. Cette amélioration est intéressante dans le cas de la prévision de la quantité de matière organique digestible ingérée $\left(\mathrm{S}_{y x}=6,05\right.$ avec le couple indice de fibrosité-cellulose Weende au lieu de 6,7 $\mathrm{I}$ avec l'indice de fibrosité seul et 6,67 avec la teneur en cellulose Weende seule).

Le fait de séparer les fourrages par cycle de croissance et par famille ne permet pas de diminuer beaucoup l'erreur de prévision obtenue avec le couple indice de fibrosité-cellulose Weende. Il est cependant intéressant de considérer séparément les graminées du I ${ }^{\text {er }}$ cycle et l'ensemble des graminées.

Cela nous conduit alors aux équations de régression suivantes :

Graminées I er cycle $(\mathrm{N}=95)$.

$$
\begin{aligned}
& y_{1}=\mathrm{I02}, 8-0,457 x_{1}-\mathrm{I}, 779 x_{2} \pm 6,99 \\
& y_{2}=\mathrm{I03}, 0-0,356 x_{1}-\mathrm{I}, 052 x_{2} \pm 3,88 \\
& y_{3}=9 \mathrm{I}, 4-0,434 x_{1}-\mathrm{I}, 603 x_{2} \pm 5,5^{2}
\end{aligned}
$$

Graminées (total) $\mathrm{N}=\mathrm{I} 28$.

$$
\begin{aligned}
& y_{1}=\mathrm{II} 7, \mathrm{I}-0,426 x_{1}-\mathrm{I}, 66 \mathrm{I} x_{2} \pm 7,94 \\
& y_{2}=\mathrm{I} 00,8-0,377 x_{1}-0,977 x_{2} \pm 4, \mathrm{I} 4 \\
& y_{3}=87,8-0,436 x_{1}-\mathrm{I}, 479 x_{2} \pm 5,70
\end{aligned}
$$

Ensemble des fourrages $\mathrm{N}=\mathrm{I} 52$.

$$
\begin{aligned}
& y_{1}=\mathrm{I0}, 5-0,701 x_{1}-\mathrm{I}, 075 x_{2} \pm 8,79 \\
& y_{2}=\mathrm{I00}, 5-0,360 x_{1}-0,982 x_{2} \pm 4, \mathrm{I} 4 \\
& y_{3}=8 \mathrm{I}, 6-0,576 x_{1}-\mathrm{I}, \mathrm{I} 73 x_{2} \pm 6,05
\end{aligned}
$$

ò̀ $x_{1}=$ indice de fibrosité $\left(0,2928 \frac{\mathrm{wh}}{\mathrm{Io}}\right)$

$x_{2}=$ teneur en cellulose brute (p. Ioo MS)

$y_{1}=$ quantité de matière sèche ingérée $\left(\mathrm{g} / \mathrm{kg} \mathrm{P} \cdot{ }^{0,75}\right)$

$y_{2}=$ digestibilité de la matière organique (p. roo)

$y_{3}=$ quantité de matière organique digestible ingérée (g/kg P. $\left.{ }^{0,75}\right)$

\section{CONCLUSION}

L'indice de fibrosité est un critère de prévision de la valeur alimentaire intéressant parce qu'il reflète bien la proportion des constituants membranaires des fourrages. Cependant sa mesure a une reproductibilité médiocre. Il est donc nécessaire de faire 6 déterminations, heureusement rapides, par fourrage. Pour pallier cette difficulté, nous avons essayé de mettre au point une autre méthode basée sur le même principe 
mais utilisant un broyeur plus gros, des quantités de fourrage plus importantes introduites mécaniquement dans le broyeur et un système d'enregistrement permettant de supprimer les variations additionnelles de la puissance électrique dues non pas au fourrage mais au moteur du broyeur lui-même. Cette nouvelle technique n'a pas permis de diminuer la variabilité des mesures ni d'améliorer les liaisons $(r=-0,826$ et $r=-0,8 \mathrm{I} 4$ entre la quantité de matière organique digestible ingérée et 1'indice de fibrosité mesuré respectivement par la ${ }^{\text {re }}$ et par la $2^{\mathbf{e}}$ méthode sur le groupe de 34 échantillons).

Nous conserverons donc la méthode décrite en I966. Ëlle peut permettre :

- aux phytotechniciens, de trier les clones ou variétés les plus intéressants en utilisant à la fois le pourcentage de feuilles et la mesure de l'indice de fibrosité. Cela a d'ailleurs été appliqué à des clones de Fétuque élevée par GILLET et JADAS-HÉcArT (I969) ; ils ont trouvé que l'indice de fibrosité était le meilleur critère explicatif de la quantité ingérée au pâturage.

- aux laboratoires d'analyses fourragères, d'améliorer la précision de la prévision de la valeur alimentaire des fourrages par rapport à celle obtenue avec la cellulose brute seule.

Reçu pour publication en mai 1971.

\title{
REMERCIEMENTS
}

Nous remercions Mme LitTRÉ pour l'aide qu'elle nous a apportée dans les calculs effectués sur ordinateur.

\section{SUMMARY}

\author{
INDEX OF FIBROUSNESS OF FORAGES ; ITS SIGNIFICATION \\ AND UTILIZATION FOR PREDICTING FORAGE FEEDING VALUES
}

The index of fibrousness of forages, measured according to a previously described method (Chenost, I966), accounts for their morphological composition (leafiness) ; their chemical composition (proportion of cell wall constituents) and their ability for being reduced into fine particles in the rumen (digestibility in nylon bags, mastication time per animal).

By combining the index of fibrousness with another simple criterium of predicting the nutritive and feeding value of forages, such as leafiness or cellulose Weende content, it is possible to improve the accuracy of the results obtained with the index of fibrousness alone or with the two criteria separately.

From I 52 forages, of different botanical families and growth cycles, we calculated the regression equations relating digestibility, dry matter intake and digestive organic matter intake to the index of fibrousness-cellulose Weende. These relationships can be improved by considering separately the grasses from the ist growth cycle and all the grasses together.

\section{RÉFÉRENCES BIBLIOGRAPHIQUES}

Chenost M., x966. L'indice de fibrosité des foins: Mesure et relations avec la valeur alimentaire. Ann. Zootech, 15, 253-257.

Chenost M., Élisabeth Grenet, Demarquilly C., Jarrige R., I97o. The use of the nylon bag technique for the study of forage digestion in the rumen and for predicting feed value. Proc. XIth Int. Grassld. Congr., 697-7oI. 
Demarquilly C., Chenost M., I969. Étude de la digestion des fourrages dans le rumen par la méthode des sachets de nylon. Liaison avec la valeur alimentaire, Ann. Zootech., 18, 419-436.

Gillet M., Jadas-Hécart J., 1969. Les facteurs de l'appétibilité. Fourrages, 41, I3I-133.

JARrige R., I96I. Analyse des constituants glucidiques des plantes fourragères. I. Fractionnement des constituants de la membrane par les hydrolyses acides. Ann. Biol. anim. Bioch. Biophys., 1, I63-2I2.

JARRige R., Thivend P., I969. Action d'une cellulase fongique sur les membranes et son in térêt pour prévoir la digestibilité des plantes fourragères. Ann. Biol. anim. Bioch. Biophys., 9, 17I-190.

Jarrige R., Thivend P., Demarguilly C., I97o. Development of a cellulolytic enzyme digestion for predicting the nutritive value of forages. Proc. XIth Int. Grassld. Congr., 762-766.

Ruckebush Y., Marguet J.-P., I963. Recherches sur le comportement alimentaire chez les ruminants. Rev. Méd. Vét., 114,833-856.

Tilley J. M. A., Terry R. A., I963. A two stage technique for the in vitro digestion of forage crops. J. Brit. Grassland Soc., 18, I04, $11 \mathrm{x}$.

Troelsen J. E., Bigsby F. W., ig64. Artificial mastication. A new approach of predicting voluntary forage consumption by ruminants. J. anim. Sci., 23, II 39-II42.

Troelsen J. E, Campbell J. B., 1969. The effect of maturity and leafinesse on the intake and digestibility of alfalfa and grasses fed to sheep. J. Agric. Sci., 73, I45-I 54 .

Troelsen J. E., et al., i97o. The effect of physical breakdown on the voluntary intake of coarse roughage by sheep. Proc. XIth Int. Grassld. Congr., 747-750.

VAN Soest P. J., I963. Use of detergents in the analysis of fibrous feeds. II. A rapid method for the determination of fiber and lignin. J. Ass. Off. agric. Chem., 46, 829-835. 